Printed in Great Britain

\title{
Studies on the Ecology of Certain Rumen Ciliate Protozoa
}

\author{
By J. MARGARET EADIE \\ Rowett Research Institute, Bucksburn, Aberdeen, Scotland \\ (Accepted for publication 3 May 1967)
}

\begin{abstract}
SUMMARY
The times taken by Ophryoscolex tricoronatus and species of the genus Epidinium to establish in the rumens of young animals were found to differ from those of other rumen ciliate species. The antagonistic relationships between certain rumen ciliates were examined in vivo and concurrent experiments were made in small vessels termed vivars which were suspended within the rumen of a sheep. A Millipore membrane separated the rumen contents from the contents of the vivars. Within these vessels it was possible to reproduce population changes first observed in vivo and to determine with certainty that in a population change where Polyplastron multivesiculatum became irreversibly dominant this had been caused by predation by Polyplastron. This organism will eliminate Epidinium, Eudiplodinium maggii, Eremoplastron and Ostracodinium from a population. The cause, factors affecting it and the ultimate effect on the population are discussed. It was not possible to cause a reversal of the population within a vivar suspended in sheep, but since the prey of Polyplastron still existed and since the reverse change was seen to occur in cattle, this problem still remains unsolved. There was also some evidence of antagonism between Epidinium and Ophryoscolex.
\end{abstract}

\section{INTRODUCTION}

Previous studies have shown that when mixtures of rumen ciliate populations from different animals are introduced into the rumens of ciliate-free sheep or cattle certain species often become dominant (Eadie, $1962 b$ ). The present work extends these observations and in particular is concerned with the relation between Polyplastron multivesiculatum (Dogiel \& Federowa) and other rumen ciliates. Investigations have also been made, incidental to the above studies, of the time taken to establish Ophyroscolex tricoronatus (Dogiel) in ruminants of different species, extending previous work on the establishment of rumen ciliate populations (Eadie, I962a).

The problems were investigated by the use of inoculations of chosen ciliate populations to lambs, kids and a calf; an in vivo method previously discussed by Eadie $(1962 a, b)$. In addition a modification of the 'vivar' technique described by Fina $e t$ al. (1962), when used in sheep, proved to be extremely useful. As discussed earlier (Eadie, $1962 b$ ) certain changes of protozoal population in lambs were irreversible, but with the aid of vivar chambers, stages of population changes could be repeatedly observed and could be compared with those seen in the animal.

Vol. 49, No. 1, was issued 28 October 1967

I 2

G. Microb. 49 


\section{METHODS}

\section{Definitions of different ciliate populations}

In a previous paper (Eadie, I $962 b$ ) the large rumen Ophryoscolecids were grouped as type A or type B according to the organisms which tended to be found together in stable populations under natural conditions. These designations have been largely retained and the organisms concerned in this paper are listed below:

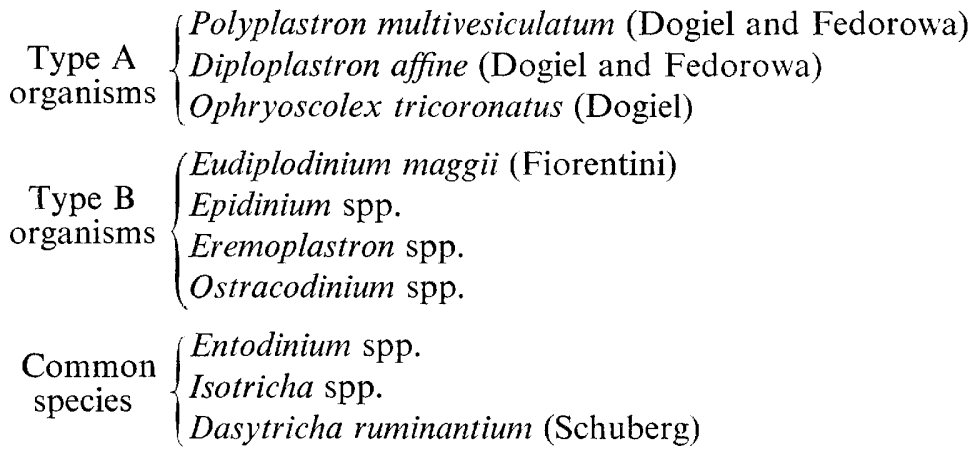

For convenience the following terminology is used through this work: (i) a 'limited' population is one in which only one or two larger organisms are present along with small Entodinium spp., e.g. E. simplex; (ii) a 'mixed' population is one, more typical of those found in nature, comprising a number of Entodinium spp., most probably both holotrich genera (Isotricha, Dasytricha) along with larger Ophryoscolecid organisms.

In the present work though the species are divided into type A and type B 'organisms'; a 'type A population' is defined as one in which Polyplastron is present either as the only large organism in a limited population or along with other type A organisms and the 'common species'. Thus (Polyplastron + Entodinium) and (Polyplastron + Diploplastron + Ophyroscolex +Entodinium + Isotricha + Dasytricha) are each type A populations, the former being a 'limited type A population' and the latter a 'mixed type A population'.

A population with the designation (Polyplastron + Eudiplodinium + Entodinium + Isotricha+Dasytricha) which can be produced experimentally would be termed a 'combined $(\mathrm{A}+\mathrm{B})$ population', since the presence of Polyplastron denotes a type A but the other large Ophyroscolecid is type B. Such combined populations tend to be unstable. The components of a limited population containing an organism of each type are mentioned by name.

There is no species restriction on the definition of a type B population so that any population containing one or more type B organisms, with or without the common species but excluding any type $\mathrm{A}$ organism, comprises a type B population.

\section{Animals and their ciliate fauna}

Young animals used for in vivo studies. Over a period of 3 years, fourteen lambs and five kids were removed from the dam and reared ciliate-free until the inoculation experiments began. Calf Z (Eadie, I962a) was also used. Some of these animals were later used as the source of ciliates for vivar experiments (see below). 
Adult animals and their ciliate fauna. Five cannulated sheep $(93,146,187,208,300)$ had mixed type A populations and four $(3,209,78,79)$ mixed type B populations. One sheep in each group $(208,209)$ was equipped with a 2 inch rumen cannula into which the vivar apparatus could be fitted. The type B population in 78 and 79 originated in cattle and included tailed epidinia and Eremoplastron. At different periods limited ciliate populations were available from the animals listed in Table I and sheep 662 was maintained completely ciliate-free. A dairy cow was used to provide a mixed type B cattle ciliate population.

Diets and management. With the exception of sheep 300, which was given a pelletted barley diet, the diets and management of the sheep were as described by Eadie ( $1962 a$ ). The kids were given a lamb diet and maintained in isolation pens similar to those used for lambs. The management of calf $Z$ has already been described. (Eadie, I962a).

Table I. Animals with limited ciliate populations available during vivar experiments

Animal and number $\quad$
Sheep
Goats

Procedure for in vivo studies

Fina et al. (1962) referred to their vivar technique as an in vivo method. To avoid confusion in the present work in vivo experiments will refer exclusively to those in which observations were made on changes within the rumen of an animal, and the vivar technique will be referred to by name.

The methods of sampling and inoculating animals without cannulae were those used previously (Eadie, I $962 a$ ), as was the examination procedure. In general the concentrated organisms from $500 \mathrm{ml}$. rumen fluid were used as a calf or sheep inoculum and that from $250 \mathrm{ml}$. fluid was used for a lamb or kid. This was varied a little with the experiment in progress, but previous work had shown that when conditions were suitable within the rumen the size of inoculum could vary within wide limits. Each inoculum was examined microscopically before use. Very occasionally an inoculum was given to adult animals via the cannula. Some limited populations were inititated by placing a few organisms in a buffered suspension directly into the mouth or cannula of the animal. The small numbers of organisms had been separated and counted under the dissection microscope. All other inocula used comprised many thousands of ciliates.

\section{The vivar technique}

The basic technique was first introduced by Fina et al. (1962) for use in cattle. The method involves the suspension in the rumen of small chambers or vivars which are fitted with Millipore membranes (Millipore (U.K.) Limited, Wembley, Middlesex) 
which are permeable to rumen solutes but impermeable to rumen ciliates. In the present work rumen ciliate populations were maintained within vivars suspended in sheep.

The vivar apparatus. Plate I, fig. I, illustrate the components of the vivar chamber. Each consisted of an ebonite vessel of Io ml. capacity at the base of which there was a Millipore membrane $(z)$ of $25 \mathrm{~mm}$. diameter held in place by the collar $(y)$. The pore size of the membranes was $0.65 \mu$. A wire gauze (stainless steel) protected the membrane from the rumen contents of the host sheep and another slightly smaller gauze lay between the contents of the vivar and the membrane to prevent any damage to the membrane while sampling. The order from exterior inwards was, therefore: wire gauze, rubber washer, membrane, rubber washer, internal wire gauze. A slight indentation in the collar accommodated these and allowed the membrane to lie perfectly smoothly. The smaller wire gauze was cut to fit exactly into the base of part $(x)$ of the apparatus and a small ridge prevented it from entering the vessel. As noted by Fina et al. (1962), provided the membrane was protected from mechanical damage it remained intact when immersed in rumen fluid.

Three of these vivar culture vessels could be suspended for as long as required in the rumen of a sheep fitted with a 2 inch cannula; Pl. I, fig. 2 illustrates how this was done.

The stem of each vivar chamber was attached to heavy rubber tubing as illustrated by PI. I, fig. 2(a). The normal cannula cap was replaced by a cap $(b)$ from which the centre had been removed. The three tubes by which the vivars were suspended were passed through holes in an ebonite disc $(c)$ and this sealed the top of the cannula when cap $(b)$ was screwed into place. The exterior end of each rubber tube was attached at $(d)$ to one arm of a light aluminium or plastic T-piece and the other arm was sealed by a rubber cap (e). At times of sampling this cap was removed and a thin polythene tube, cut to a suitable length to reach the base of the vivar, was passed in. Withdrawals or additions were made with the aid of a $10 \mathrm{ml}$. glass syringe, and the contents of the vivar could first be gently mixed by several movements of the syringe. While this was in progress nitrogen from a balloon was allowed to flush the system through the stem $(f)$ of the T-piece. Some time before sampling the syringe had been attached to the sampling tube and both had been filled with nitrogen and the tube sealed until the sample was to be taken. After sampling, the stem of the T-piece acted as a gas outlet by being connected as shown $(g)$ to a narrow polythene tube, the other end of which was passed through the cap of an $8 \mathrm{ml}$. polythene centrifuge tube $(h)$ and under the surface of a small volume of water held there. This prevented the entrance of atmospheric oxygen. Finally, all three tubes were tied with string until they were in the position shown by $(i)$. Thus the extension of the apparatus from the sheep's cannula was decreased to a minimum, and the sheep could feed and move freely in its pen and did not appear to be affected by the apparatus. The plastic sheet $(j)$ was placed over the cannula as shown to decrease contamination from any rumen fluid which leaked on to the sheep's wool round the edge of the cannula.

When suspending the vivars in the rumen it was essential for ease of sampling to make sure that they were spaced slightly apart. A length of fence wire covered with plastic was bent into a hook at the end and used to push each vivar into the rumen at the correct angle. Once they were placed in this way they very rarely became entangled.

Preparation of ciliate-free, centrifuged, rumen fluid. Rumen fluid obtained from the 
ciliate-free cannulated sheep was centrifuged for $5 \mathrm{~min}$. at $630 \mathrm{~g}$ and the supernatant fluid used.

Preparation of washed suspensions of protozoa. The previously described method with separating funnels was used (Eadie \& Oxford, 1955) but with the bicarbonate buffer described by Abou Akkada \& Howard (1960). Suspensions from smaller volumes of rumen fluid were obtained by decantation in $100 \mathrm{ml}$. boiling tubes.

Additions to vivar. Pure rice starch was obtained from Reckitt \& Sons, Ltd., Hull. Two ground-nut meals (GN. I5, GN. I7) were used; these were chosen because of their high protein content and low nitrogen solubility in water ( $15 \%$ and $8 \%$ respectively, of the total nitrogen was soluble in water.) Ground-nut meal GN. I 5 was known to have a 'very high' aflatoxin content (2.5 p.p.m. of aflatoxin). Acetone-dried barley-leaf protein as prepared by Duckworth \& Woodham (I96I) was also used. It had an even lower nitrogen solubility in water than had meals GN. I 5 and GN. I7.

Routine procedure. The Millipore membranes were soaked in buffer before being fitted into the vivar chamber as described above. Then $5 \mathrm{ml}$. of ciliate-free centrifuged rumen fluid and $5 \mathrm{ml}$. of the chosen ciliate suspension were added by pipette to the chamber. The ciliate suspensions were thoroughly mixed and pipetted rapidly. Frequently a small amount of rice starch (IO-I5 mg.) was also added at this time, the amount used being gauged by the number and state of fill of the ciliates. While a gentle flow of nitrogen was being passed through the rubber tubing (PI. I, fig. $2(a)$ ) the vivar was carefully fixed to the end. The whole assembly was then carried to the sheep, any subsequent changes to the contents being carried out in situ with the aid of a sampling tube and syringe as described.

The components of the suspension, and the number and degree of fill, of the organisms, could be altered as required, but the number of controls per experiment was necessarily limited since only six vivars could be run at one time (three per host sheep). Care was taken to mix the contents of the vivar immediately before withdrawal of samples in order to use comparable numbers of protozoa. The contents of the vivar were regularly sampled and rice starch suspensions added as required. It was necessary to judge from the appearance of the ciliates before feeding the amount of starch required, as this varied with the number of organisms and the time since the vivar had started. The barley-leaf protein and ground-nut meals were similarly added as suspensions.

\section{RFSULTS}

\section{IN VIVO EXPERIMENTS}

\section{Observations on the establishment of Ophryoscolex tricoronatus}

This organism, although established, had subsequently died out in several sheep (Eadie, $1962 b$ ). However, the species was again established in three cannulated sheep at the Institute by inoculation from animals brought in from other areas and it was later established along with small Entodinium spp. in isolated sheep 661.

Since there had been some indication that Ophryoscolex was slow to establish when given to young lambs in a mixed inoculum Eadie (1962a), further inoculation experiments were made with both ciliate-free lambs and kids. In all cases the rumen conditions would be expected to be suited to ciliate development. The time taken to establish the organism when using a mixed type $\mathrm{A}$ inoculum including Ophryoscolex, was compared with that when Ophryoscolex was the only type A organism present (sheep 66I). 
It is clear from Table 2 that except in lamb 56 Ophryoscolex regularly failed to become established after the first few inoculations into a young animal. This was true whether Ophryoscolex was the only large ciliate in the population or was a component of a mixed population. Though the first inoculum was successful in lamb 56 this animal was possibly atypical since it was not well at the time of the first inoculation, and its ciliate population died while the animal was being treated for pneumonia. It required two further inoculations to re-establish the organisms. Other than in lamb 56 the population developed at a slightly earlier age in the kids than the lambs, as had been

Table 2. The establishment of Ophryoscolex populations in sheep and goats

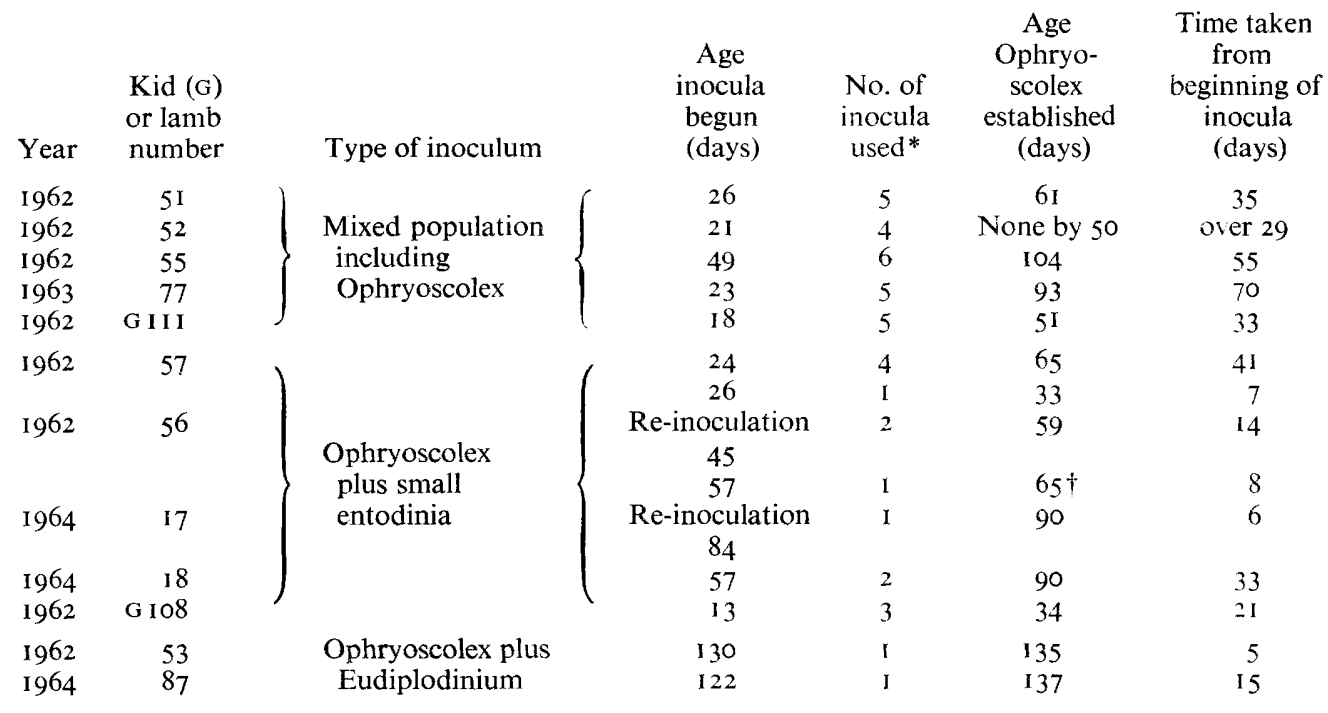

* The interval between inocula was between 7 and 10 days except in 77,17 and 18 - see text.

$\dagger$ One active organism was seen at 65 days, but Ophryoscolex was not seen in subsequent samples until after re-inoculation.

suggested by previous observations (Eadie, 1962a). The regular inoculations were discontinued for intervals of 2 weeks and 4 weeks in lamb 77, but Ophryoscolex did not develop. In lambs 17 and 18 a month interval was left after the first inocula, but second inocula were necessary. These observations proved that the Ophryoscolex was not already established and merely developing more slowly after the first inoculum than components of other limited populations. As seen in lambs 53 and 87, the time required after inoculation and the number of inocula necessary tended to be less at a greater age, and in the isolated animal 66I a population was established at 11 months of age after one inoculation with not more than 27 organisms. Nevertheless, Ophryoscolex was still observed to die out in older animals and could not always be reestablished (see later). Shortened, 'stumpy', caudal processes were frequently noted in the Ophyroscolex when a population was first becoming established.

\section{Observations on the development of other species in young animals}

Type A organisms other than Ophryoscolex have been shown to establish very rapidly in young animals. When rumen conditions were suitable for ciliates, a first inoculum at 3 weeks of age usually resulted in establishment (Eadie, 1962a). 
When inocula of mixed ciliates of type B from cattle were given to two lambs 78 and 79 at 5 weeks of age, all but the tailed species of Epidinium were readily established. Epidinium ecaudatum developed I week after the first inoculation, but the tailed species of Epidinium were only seen at 4 and 5 weeks after second inoculations. It was later noted that Ostracodinium, a genus more typical of cattle than sheep, had died out of both lambs--by 6 months after the first inoculum in 78 , and by 9 months after the first inoculum in lamb 79. In a third lamb given a mixed type B population as inoculum all Epidinium species failed to establish. Dasytricha ruminantium is the only other species in which variation in establishment time has been noted.

\section{Establishment of limited populations containing Ophryoscolex and one other large Ophryoscolecid}

Ophryoscolex plus Polyplastron. It proved quite easy to establish and maintain a population containing these organisms and small entodinia in both a kid (GI08) and a lamb (8I). (see Table 3). This limited population in kid GI08 was of value in vivar experiments on antagonism (see later).

\section{Table 3. Establishment of apparently stable limited ciliate populations* in young animals which were initially ciliate-free}

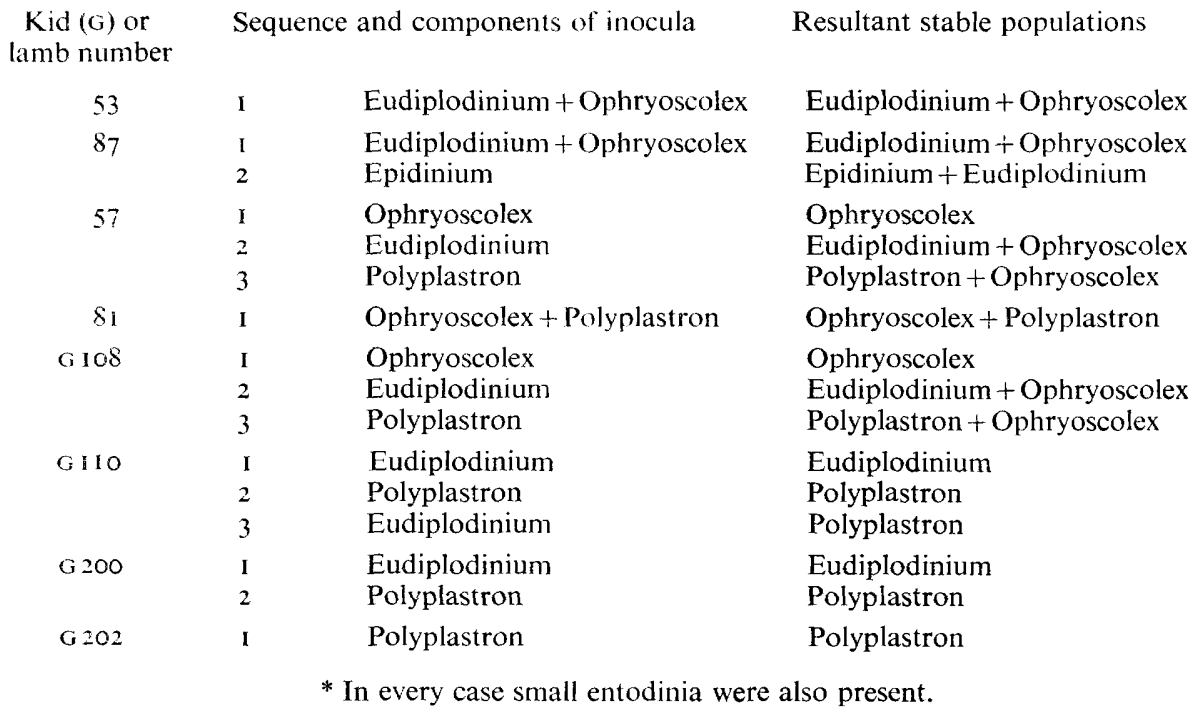

Ophryoscolex plus Eudiplodinium. It was of interest to determine whether Ophryoscolex, frequently found in a type A population, and Eudiplodinium, an important component of the type B population, would develop as the only large Ophryoscolecids in a rumen population. This proved possible in sheep 53 and 87 using a mixture of the two protozoa from sheep $66 \mathrm{I}$ and 95 as inoculum. The mixed population remained in 53 for $3 \frac{1}{2}$ years but at this point Ophryoscolex dwindled in number and disappeareda change similar to those noted below in mixed type A populations. The same limited population was successfully established by inoculation of the separate species into lamb 57 and into kid G 108 (see Table 3). 
Ophryoscolex plus Epidinium. It was not possible to establish these two genera in the same animal, whether alone or in a mixed population; this problem is being further investigated. As an example, when Epidinium was inoculated into sheep 87 following the establishment of Ophryoscolex and Eudiplodinium (above) the Ophryoscolex gradually disappeared and Epidinium developed along with Eudiplodinium. So far Epidinium consistently appears to develop in preference to Ophryoscolex when the two organisms are mixed in vivo.

The disappearance of Ophryoscolex from mixed type A populations. The three cannulated sheep used to establish Ophryoscolex in an already established type A population were $I \frac{1}{2}, 2 \frac{1}{2}$ and 10 years old. The oldest animal was one from which Ophryoscolex had previously disappeared (Eadie, I $962 b$ ), yet the organism re-developed and was present in fair numbers when the sheep was killed 4 months after the inoculation. A second animal was killed $I \frac{1}{2}$ years after inoculation; Ophryoscolex was still found to be present. In the third and youngest animal the Ophryoscolex dwindled in numbers and were not seen after I7 months from the inoculation. These observations would indicate that the disappearance of Ophryoscolex from a population is not directly related to the age of the animal.

The instability of Ophyroscolex in ciliate populations was further examined by using sheep 146 . This animal had a mixed type A population which had previously included Ophryoscolex, but though regularly inoculated with Ophryoscolex from the isolated sheep 66I the species was not re-established. To investigate this further the following two experiments were carried out.

(i) Lamb 8I with a well-established population of Polyplastron and Ophryoscolex was inoculated with rumen material from sheep I 46 . The other ciliates from sheep I 46 developed in lamb 8I yet Ophryoscolex remained numerous for Io weeks. When numbers of Ophryoscolex later decreased the population was increased again by inoculation. It seemed that there might be some relationship between Diploplastron and Ophryoscolex, but there appeared to be no direct or rapid effect of the sheep I 46 rumen inoculum per se, yet over the same period of time inocula from sheep 66I did not re-establish Ophryoscolex in sheep I 46.

(ii) This experiment was done with twin lambs 17 and 18 , in which Ophryoscolex had been established as the single large species by inoculation (Table 2) and had developed well. At 125 days of age lamb I7 was inoculated with a mixed type A population from sheep 208 in which Ophryoscolex had been established for several years and lamb I 8 was inoculated from sheep 146 in which Ophryoscolex had repeatedly failed to establish. In other respects the ciliate populations were similar. In this experiment it was lamb I7 which showed a great decrease in Ophryoscolex but, though fluctuating in number, the organisms remained. At 223 days of age, when the experiment was terminated, Ophyroscolex was still present in both lambs. Once again there was no indication that the 146 population in itself could remove Ophryoscolex from another animal.

\section{Further in vivo investigation of the antagonism between type $A$ and type $B$ populations}

Inoculation experiments were made partly to confirm and extend previous findings and partly to make comparisons with the vivar experiments (see later). Type A populations had become dominant in both sheep and cattle rumens but a type B population 
was seen to become dominant in calves F, G and 35 (Eadie, 1962 b). Large Eudiplodinium organisms, never observed in sheep, were associated with this latter change and also with extended periods of time in which organisms of both types were together in cattle. Thus the presence of large eudiplodinia might be significant in the reversal of the antagonism. The previous findings suggested that the type A organism Polyplastron and the type B organism Eudiplodinium were the only mutually antagonistic species, but studies of the relationship between Epidinium and Ophryoscolex may cause some revision of this view. Ophryoscolex was not present in the type A populations used in 1962, (Eadie, 1962 b), hence the present interest in this species. Similarly changes of population in goats had not been examined.

Cattle. Calf $\mathrm{Z}$, which had been used as an isolated animal (Eadie, $1962 a$ ), was inoculated with a mixed type $B$ population from a cow. When this population had been established a type A population was inoculated and, after 3 weeks in which both were present, type A became dominant. When re-inoculated with a type B population calf $Z$ showed an effect similar to that previously observed in cattle (see above); that is, an extended period in which both Polyplastron and Eudiplodinium were seen in fair numbers and in which Eudiplodinium of an unusually large size were present amongst the re-established population. The other type $B$ organisms did not become re-established. After 2 months with the combined $(A+B)$ population type A again became dominant. Ophyroscolex was included in the type $A$ population but only very small numbers were ever seen.

Goats. In three animals G 108, G110 and G200 the dominance of Polyplastron (type A), when inoculated from the isolated sheep 683, was demonstrated (see Table 3). In all three Eudiplodinium was the only type B organism initially present but in goat G I08 Ophryoscolex was also present initially. The Ophryoscolex remained along with Polyplastron after the change. The time taken to complete the change in population was 9 , I I and 12 days. This contrasts with the longer time taken in some calves (e.g., calf $Z$ above). In two of the goats unusually large Eudiplodinium organisms similar to those noted in calf $Z$ were seen during the period when a combined type $(A+B)$ population was present (see PI. 2, figs. I, 2). The Polyplastron also tended to be large and were often microscopically extremely dense just before the change-over-a feature of the antagonism frequently noted (Pl. 2, fig. 3). After the change some Polyplastron remained large for some time and were used in vivars (see later). Neither largenor normal-sized eudiplodinia were ever observed again following inoculations of large numbers of eudiplodinia to goat G110.

Thus the population in goats displayed features of the antagonism intermediary between sheep and cattle. As in sheep, Eudiplodinium did not become re-established even temporarily but large Eudiplodinium were observed, at the time of the change. In other respects, for example, the numbers of large bacteria such as Oscillospira which develop in the young ciliate-free animal, kids seemed similar to lambs (Eadie, $1962 b$ ).

Sheep. As was expected, when Polyplastron and small entodinia from the isolated sheep 683 were inoculated into lamb 57, which had a population of Eudiplodinium and Ophryoscolex, the Polyplastron became dominant and all Eudiplodinium disappeared (Table 3); as in the goat G 108 the Ophryoscolex also remained but no large Eudiplodinium were observed. This confirmed previous observations. As the previous experiments indicated, very dense, frequently dividing, and quite large 
Polyplastron were associated with a population change (Pl. 2, fig. 4; see Eadie, I962 b, for lamb 222). Organisms in which internal structures such as the two large skeletal plates were clearly visible (see Pl. 2, fig. $6 b$ ) were more typical of Polyplastron from animals on the diets used here.

In an effort to induce the development of large Eudiplodinium organisms in a sheep, two inocula of $100 \mathrm{ml}$. and $200 \mathrm{ml}$. of whole rumen fluid from calf $\mathrm{Z}$, when a combined type $(A+B)$ population was present and large eudiplodinia were conspicuous, were given to a sheep. Before the inoculation only Isotricha, Polyplastron and Entodinium were present in the sheep. The inoculations led to the development of Diploplastron, Dasytricha, and even a few Ophryoscolex which had been very infrequent in calf $Z$. However, no Eudiplodinium either large or small were observed. Similarly, a smaller inoculum into another sheep with a type A population failed to establish the large Eudiplodinium, even temporarily, though Diploplastron of type A was added to the population.

With one exception, experiments to date have confirmed the previous findings that a type A population would readily and irreversibly remove type B organisms from a sheep rumen fauna; the exception was with sheep 209. Though this animal was placed between, and in contact with, two sheep with mixed type A populations for over 9 months, its type B population was retained. Even after one small inoculum (about 40 organisms) and two larger inocula of Polyplastron plus small entodinia (concentrated from $175 \mathrm{ml}$. and $600 \mathrm{ml}$. sheep 7I rumen fluid) the Eudiplodinium of type B population remained. Though Polyplastron were seen for a short time large Eudiplodinium were never observed. Three weeks after Polyplastron was first seen Epidinium disappeared. Two other sheep which had earlier received a type B population direct from sheep 209 showed a typical change of population, in one case due to accidental contamination with what must have been very few organisms. It appeared that sheep 209 might have shown an animal difference but unfortunately the animal died of pneumonia before further experiments could be completed; at the time of death Polyplastron and Eudiplodinium were both present in the population. The rumen fluid had on occasions been as low as $\mathrm{pH} 5.5$, and the postmortem examination indicated that the animal had been suffering for some time from subclinical pneumonia. It is possible that these factors may have affected the population.

\section{VIVAR EXPERIMENTS}

It was clear from the present study and previous work that experiments in vivo were not suitable to determine the cause of the antagonism between type A and type B populations, but could only demonstrate under what conditions it existed. The chief object of vivar experiments was to determine the cause. The vivars had the great advantage that both populations could be examined together in a closed system, unlike the case in sheep in vivo, for example, where re-inoculated type B organisms have never been seen.

\section{Maintenance of normal cilates within a vivar}

Preliminary experiments were made with different concentrations of ciliates in the initial inoculum and variations in the frequency and amounts of rice starch fed. The numbers of ciliates which could be used varied over a wide range, and naturally 
varied with the components of the population: later counts of fixed inoculum suspensions showed that in general, in a satisfactory run, the concentration in the vivar at the onset of the experiment had been 2-5000 large Ophryoscolecids $/ \mathrm{ml}$. The organisms were usually fed with rice starch twice daily, the amount ranging from 10 to $25 \mathrm{mg}$., depending on the numbers and species present and the length of time that the vivar had been running. The rice starch was always added as a suspension in buffer, the volume usually being the same as the last vivar sample taken. The initial rate of starch utilization was noticeably greater than at a later stage and the gauging of the first overnight feeding was particularly important. However, with experience, organisms could be maintained in an apparently normal active state for 4-6 days. Beyond that time, it seemed inadvisable to rely on the results because the ciliates, though still alive, appeared to be less healthy, and the background bacteria seemed to become more prominent.

The Millipore membrane completely prevented the passage of ciliates in either direction, and since the centrifuged rumen fluid was taken from a ciliate-free animal there was no possibility of ciliates being added in that way. The following populations could be maintained for several days: a mixed type A population, a mixed type B population, Eudiplodinium plus Ophryoscolex, Polyplastron plus Ophryoscolex, also Polyplastron, Eudiplodinium and Ophryoscolex alone save for small entodinia. It was noticeable that the proportions of certain species within a mixed population in a vivar tended to change with time. Thus there seemed to be some competition between large entodinia (Entodinium bursa Stein) and Diploplastron affine in the mixed type A population though there was no sign of one population rapidly eliminating the other. Similarly in a mixture of the two, the numbers of Ophryoscolex were not maintained as well as Eudiplodinium, but again division rate and competition for rice starch under the restricted conditions of the vivar might account for this. When only Ophryoscolex and entodinia had been inoculated into a vivar the proportion of Ophryoscolex with short 'stumpy' caudal processes rose, even after only $\mathrm{I} 2 \mathrm{hr}$. (e.g. from less than Io \% in the inoculum to an average of over $60 \%$ in the three vivars of one run). This might be compared with the occurrence of these forms in vivo in a young animal or isolated animal when Ophryoscolex was first becoming established.

Though it was possible to maintain Ophryoscolecid ciliates which ingested rice starch, the vivar technique was not so satisfactory for the holotrich organisms, especially Dasytricha, which tended to become abnormally empty within a few days. It seemed that the diffusible sugars from the host sheep did not become available in sufficient quantity; though clearly some nutrient was available or Dasytricha which does not ingest starch granules would rapidly have died.

\section{Bacterial development within a vivar}

The Millipore membrane would exclude all but the smallest bacteria. On a number of occasions, Gram-stained films of the vivar contents were examined. These indicated that for any one controlled run (that is three vivars in one sheep) the bacterial picture was similar in each vivar. During an experiment an increase in small Gram-positive rods was observed, especially when a ciliate population had been allowed to die within the vivar, but there was no strikingly abnormal development of bacteria. A control vivar without ciliates did not show the same increase in small Gram-positive rods until starch was added, showing that the starch within the dead ciliates had possibly 
stimulated the rod development. On the whole, therefore, it could be assumed that over the first few days bacterial conditions in the vivar were not unlike those within the rumen, though many of the large bacteria had been removed by the light centrifugation of the rumen fluid added to the vivar. The bacterial picture was dependent on that of the centrifuged rumen fluid used initially and small amounts of this were always kept for further examination in case there was any unusual development within the vivar.

\section{The effect of host sheep with different types of population}

It was initially believed that a diffusible material similar to the toxic biotic substances which affect certain algae (Rice, I954; Proctor, 1957) might be the cause of the observed antagonism between type A and type B populations. Thus the viability of a type B population within a vivar when suspended in a sheep with a type A population was compared with that when a vivar was suspended in a host sheep with a type B population. The ciliates within the vivar appeared to be unaffected by the host sheep population. Since the Millipore membrane would allow the passage of a diffusible material it was assumed that antagonism between type $\mathrm{A}$ and $\mathrm{B}$ populations must be due to a more direct effect.

\section{The effect of mixing populations of type $A$ and type $B$ within a vivar}

When populations of type A and type B were mixed within a vivar the antagonism observed in vivo in sheep was reproduced, i.e. dominance of type A Polyplastron. The type B organisms had generally disappeared by $I-2$ days after the two populations were mixed, and it was immaterial which population was placed in the vivar first. Even I 2-24 hr establishment of one population did not alter the speed of the change. When Polyplastron was about to become dominant the organisms were often observed to be very similar to those seen in vivo at the time of a population change, very densely filled organisms, sometimes quite large, with division frequently seen (see Pl. 2, figs. $3,4,5)$.

\section{Procedure used in further experiments}

To make control and repeat experiments a standard procedure had to be used. Because of the problems of food competition and the greater difficulty of assessing food requirements in a mixed population it proved more convenient to use a limited population in most experiments. Changes using mixed type A populations were made but unless the change was effected rapidly it was not easy to assess the effect produced by the rather large numbers of background ciliates. The presence of a few small entodinia in the washed suspensions used from limited populations proved a great advantage, since they indicated, by their state of fill, the excess starch which had been available. With practice it became easy to assess and adjust the concentration of a population to be used for an inoculum; suitable suspensions could be chosen by examination of comparable volumes under the microscope. Where controls from different suspensions were being used a rough count was made on several drops of equal volume. As speed was vital these assessments had to be made more rapidly than would be possible with an accurate counting technique. However, in any one experiment there appeared to be good repetition and slight variations in subsequent runs were a useful check of the reproducibility of an observation. Usually, the volume of fluid removed at 
sampling was replaced by the buffer of the food suspension. As the capacity of a vivar was small, it was not possible to do any accurate counts on the population during a run, and because of unknown changes of volume through diffusion such counts would be of little value. However, since the ciliates could not escape from the vivar, changes in relative proportions could easily be recorded. The state of fill of the organisms could also be judged quite easily and this was of experimental interest as well as being necessary in the assessment of food requirements. In general each vivar run was started at mid-day and any additional ciliate organisms were added $12-\mathrm{I} 8 \mathrm{hr}$ later, when the first population had become established. When the population change followed $\mathrm{I}-2$ days later, the organisms were generally left to return to 'normal', i.e. a size and microscopic density similar to that normally found in vivo. Thus an average run lasted about 5 days, which was within the time limit of the technique. The contents of each vivar were examined from 1 to 3 times/day and at least three vivars were used for each run.

\section{Investigation of the nature of the antagonism}

Since the antagonism occurred when the two populations were in direct contact it seemed that the cause might be either food competition, as shown between ciliate genera in activated sludge by Brown (1964), or predation. As mentioned above, during a population change the polyplastrons became so dense that it was impossible to distinguish the contents under the light microscope (as in vivo experiments). These organisms returned to 'normal' I-2 days later. In contrast, when cannibalism was occasionally noted in Polyplastron from sheep which had been without food overnight ( $12 \mathrm{hr}$ ) the ingested organisms could be seen quite easily (Pl. 2, fig. 6). In any case the observation of what Lubinsky (1957) called 'accidental predation' would not be proof that predation was the cause of the antagonism. Ingestion could be seen very occasionally in a natural ciliate population, for example, holotrichs and entodinia within Polyplastron, yet these ingested species were not eliminated from a population. Similarly Lubinsky (1957) recorded Ophryoscolex within Polyplastron, although he also pointed out that Dogiel (1947), who must have studied many thousands of rumen ciliates, stated that predation did not occur in the larger Ophyroscolecids. Thus to prove that selective predation was the cause of the population changes it was essential to eliminate the superficially more probably cause, namely food competition.

\section{Initial experimental evidence against food competition}

In several experiments, at the time type $\mathrm{B}$ organisms had been eliminated from a mixture of type A and type B populations, the type B organisms living alone in a control vivar were seen to be well filled and active. This was observed even where the quantities of rice starch had been adjusted so that considerably less was given to the control than to the vivars containing both types of organism.

After a change of population the small entodinia were often well filled. This would indicate that the starch had not been completely used up by the type A organisms and that some should therefore have been available for type B organisms as well as entodinia.

The occasional appearance of ciliate fragments during a change-over indicated some deaths within the vivar. However, if the elimination of type B organisms was entirely due to death by starvation during a vivar run, then many fragments of the organisms should have been visible; this was not the case. If food competition had 
occurred the only other explanations were that these fragments were ingested by Polyplastron or had disintegrated rapidly. An experiment was therefore made to to examine these possibilities. Active polyplastron were placed in a vivar containing a suspension of type B organisms previously allowed to die by starvation in a vivar. There seemed to be no decrease in the number of fragments where Polyplastron was present, as compared with a control without. Also the fragments remained clearly distinguishable over 5 days.

Thus it seemed clear that competition for food, in the form of starch, was not the primary cause of the disappearance of the type B organisms. The other suggested cause, predation, was confirmed by the observations below.

\section{Direct observation of predation and cannibalism within a vivar}

During repeated examinations of vivar contents at the time of a change-over of population it was possible, very occasionally, to see ingested organisms; on one occasion numerous polyplastrons were seen to have ingested type B organisms. Although the actual process of ingestion was not observed, one ingested organism was seen alive for several minutes. However, within $20 \mathrm{~min}$. the polyplastrons began to develop the typical extremely dense appearance, after which the contents could not be distinguished. This rapid change explains the difficulty of determining the cause in vivo.

The fact that the mouthparts of Polyplastron are extremely elastic and strong was illustrated by a form of cannibalism in Polyplastron which occasionally followed a population change in a vivar. As illustrated in P1. 2, fig. 7, though Polyplastron is fairly rigid and is not easily distorted mechanically, it was considerably misshapen. A few polyplastrons were seen with extended mouthparts which were attached to other polyplastrons as if by suckers. This particular form of cannibalism has not been seen in vivo; it was possibly a result of the confined conditions within the vivar. Gelei (I925) noted a similar chewing effect in cultures of cannibalistic Stentor.

Having determined the most probable mechanism of the antagonism between Polyplastron and type B organisms further experiments were made to investigate the factors involved. In all experiments, though predation was known to be the chief cause of the antagonism, it was essential to beware of masking the result of a run by an increase of food-competition effects. Unless an effect was exceptionally striking it was necessary to do a number of controlled repeat experiments before drawing the conclusions cited below.

Observations on factors affecting the antagonism between certain protozoa, based on vivar experiments. The observations may be recorded in the following subsections.

\section{General observations on predation in Polyplastron}

I. It was immaterial which population type was established first in a vivar or the two could be mixed in vitro immediately before inoculating the vivar. Thus there seemed to be no question of 'conditioning' the vivar contents. Provided the same sources of ciliate populations were used the change in population (that is, the disappearance of type B organisms) occurred after the same time interval, usually between 24 and $48 \mathrm{hr}$.

2. As in sheep, the smaller type B organisms were removed first and Epidinium before Eudiplodinium. The latter was always the last to be removed. 
3. As in the in vivo work (Eadie, $1962 b$ ), changes of population from type B to type $A$ in vivars were often followed by a decrease in the numbers of Polyplastron.

\section{Cannibalism and its comparison with predation}

4. Cannibalism was observed under two conditions. (a) When Polyplastron of a limited type A population had been left overnight in a vivar and had become moderately empty. This form of cannibalism was similar to that occasionally seen in vivo when the protozoa had been some time without food (P1. 2, fig. 6) and would agree with Dawson's (1919) observation that cannibalism occurred when food was beginning to be depleted. (b) On occasion, immediately after the removal of a type B population or when very few type B organisms still survived, a few chewed misshapen polyplastrons were conspicuous amongst the well-filled organisms which remained (Pl. 2, fig. 7).

5. An experiment in which moderately filled Polyplastron were fed on mixed type B organisms and the control was left without food showed that, whereas the predators lived for $1-2$ days, the polyplastrons alone, though showing signs of cannibalism, died in a manner typical of starvation. Thus cannibalism was less effective nutritionally than predation.

\section{Ciliates not removed by Polyplastron}

6. Small entodinia were not removed even when they were the only organisms along with active cannibal polyplastrons.

7. Observations made on Ophryoscolex in sheep and goats were confirmed, i.e. Ophryoscolex would remain, when present in the original mixture, regardless of whether it was initially from a population with Eudiplodinium (type B) or Polyplastron (type A). Ophyroscolex was decreased in numbers, but this possibly was due to food competition or slower division rate and occurred in a vivar when Ophryoscolex was maintained with species other than Polyplastron (see p. 185).

8. When the mixed type A population from sheep 146 was used in vivars it removed a mixed type B cattle population, and also Eudiplodinium from a mixture of Eudiplodinium plus Ophryoscolex. Thus for the duration of a run, Ophyroscolex remained in a vivar along with the sheep I 46 population, and this at a time when large inocula of Ophryoscolex placed directly into sheep I 46 via the cannula did not establish the organism (see p. 182).

\section{Conditions under which Polyplastron did not remove type B organisms}

9. Starvation, rather than stimulating predation, appeared to prevent it. Moderately full, apparently healthy polyplastrons were necessary. An experiment to compare moderately full organisms immediately after removal from a sheep and nutritionally similar ones from the same population but kept for several days in a vivar, indicated similar predatory activities. However, when the predatory activity of Polyplastron starved in a vivar was compared with moderately full organisms from a sheep, the starved organisms did not show any sign of removing a type B population. Also, there was some indication that very empty polyplastrons, on the point of death, became unusually non-selective and would ingest fragments of dead ciliates, thus contrasting with normal or predatory polyplastrons which did not ingest dead remains (see p. I 88).

10. Other than starvation and the experiments with sheep 209 (see below), the only observed cause of failure of a change-over was the absence of a minimum number of 
polyplastrons. This was shown by inoculating into a vivar containing a type B population a small number of polyplastrons (about 30), and comparing this with the effect of the usual inoculum of some thousands. The small number of polyplastrons lived and were clearly predatory, but did not effect the change within the 4 days of experiment. However, there was no sign of a reversal to type B only, nor were any large Eudiplodinium observed. The minimum number of polyplastrons required could not be stated categorically because it depended to a certain extent on the predatory activity of the particular type A population used.

\section{Variations in the predatory activity of Polyplastron}

II. There was some indication of variation in predatory activity of Polyplastron from causes other than starvation. Predatory activity could be judged by comparing the speeds of removal of a given type B population, or the speed of apparent increase in numbers of Polyplastron relative to the Eudiplodinium, should complete removal not occur. As mentioned (p. 183) there was a tendency for larger polyplastrons to be present during a change-over, in vivo or in a vivar. In goats GIO8 and G IIo larger polyplastrons remained in the animal for I-2 weeks after the change-over and in several vivar experiments these organisms showed more rapid predation than control organisms from sheep 683 .

I2. There was some evidence of a similar increase in predatory activity when polyplastrons which had already removed a type B population in a vivar were compared with polyplastrons direct from a sheep. This increase in activity was less convincing than that mentioned in section I I above, but in several experiments there appeared to be a more rapid increase in the proportion of Polyplastron relative to the type $B$ organisms. Also, when polyplastrons given a second inoculum of type B organisms were compared with polyplastrons which had been maintained alone in the vivar, those which had previously removed a type B population were more active predators.

I3. When twelve large-sized polyplastrons from a double 'take-over' (section I2 above) were inoculated into a ciliate-free lamb, small polyplastrons developed. These later died out, but the significance of this is not known.

I4. Large size is often associated with predation and cannibalism in ciliates (Giese, 1938; Giese \& Alden, I938; Dawson, 1919). Some organisms, for example, Blepharis$\mathrm{ma}$, Stylonychia and Oxytricha, appear to get progressively larger with an increase in size of prey (Giese, 1938; Padmavathi, 1961). This was not the case with Polyplastron. When polyplastrons in a vivar were fed small Eremoplastron, followed by larger type B organisms, the polyplastrons did not become progressively larger, and as in other experiments they became normal (that is small again and less dense) $I-I \frac{1}{2}$ days after cessation of feeding.

I5. Unusually large polyplastrons from the barley-fed sheep 300 were considerably more active predators than average-sized control polyplastrons. The change-over with the former could occur within I2 hr, but with the latter took I-2 days (see section I above).

\section{Predatory activity in the presence of an insoluble protein}

I6. Both ground-nut meal (meals GN. I5 and GN. I7) and barley-leaf protein were used to see whether they acted as a sparer of type B organisms. These two protein materials were very insoluble in water so that they remained particulate within the 
vivar. Both materials were readily ingested by the ciliates, and though ground-nut meal GN. I 5 had a high aflatoxin content there was no sign of toxicity for the protozoa. There was an indication that predation was slightly slowed down when the proteins were available, but it was not prevented. There was a very clear indication that the presence of protein increased the rate of utilization of rice starch during a population change, since organisms with available protein became less well filled than the controls and had to be fed with more rice starch than the latter. This was also true whether Eudiplodinium, Ophyroscolex or Polyplastron was the only large Ophryoscolecid in a vivar.

\section{Attempts to initiate dominance by type $B$ organisms including experiments with sheep 209 population}

17. Attempts were made to induce a change of population from type A to type B organisms by the alteration of conditions (such as relative numbers and state of fill) in the limited populations generally used. These attempts were unsuccessful and no large Eudiplodinium were ever seen (see p. 184).

I8. The vivar technique had not been perfected when large Eudiplodinium were present in calf $Z$. Thus the nearest approach to a dominant type B population was that in sheep 209 when inocula from sheep 7 I did not cause a population change from type B to type A. (p. I 84). Vivar experiments were made with material from sheep 209 and $7 \mathrm{I}$, but the very large number of medium entodinia (e.g. E. caudatum and $E$. longinucleatum) in vivar inocula from sheep 209 made repeat experiments to assess the food requirements of the organisms essential, but unfortunately the sheep died before enough experiments were completed. However, the following experiments were made. (a) In one vivar run of the three completed with sheep 209 type B organisms and type A organisms from sheep 7I, Polyplastron became dominant; that is, all type B organisms disappeared. In the other two runs the two populations lived together over the 5 days of the vivar run. The significance of the last two runs is difficult to assess because of the possible inadequacy of the food supply available to the Polyplastron due to competition from entodinia (see above). However, there was never any sign of type B organisms becoming large or dominant. (b) Since sheep 209 was a host it was possible to carry out a vivar run in that animal and to compare it with a control run in sheep 208. Again sheep 7I provided the type A population and in this case centrifuged rumen fluid from sheep 209 was used in the vivars in sheep 209. The object was to determine whether the factor preventing Polyplastron from sheep 7I from becoming dominant in the rumen population of sheep 209 would have any effect on the vivars suspended in that animal. This experiment took place just before the death of sheep 209 when both types of organisms were seen in that animal. Type B organisms were not removed from either set of vivars, large Eudiplodinium did not develop, and over the 5 days of the vivar run there was no indication that the polyplastrons suspended in sheep 209 were any less active than those in sheep 208. Thus, although type A organisms did not become dominant in sheep 209, a change of population did take place in one vivar, so that there seemed to be no direct effect of the sheep 209 population. The slowness of change in the other vivar experiments may have been a reflection of the state in sheep 209 in vivo, but it may equally have been due to a decrease in the predatory activity of Polyplastron through food shortage. The polyplastrons from sheep 7 I were active in other experiments made concurrently. 


\section{The effect of mixing Ophryoscolex and Epidinium}

19. Vivars were used to examine the relationship between Ophryoscolex and Epidinium, but over the period of a vivar run there was no indication of antagonism. A decrease in numbers of Ophryoscolex relative to Epidinium was noted but this was similar to that observed when Ophryoscolex was in a population with Eudiplodinium or Polyplastron.

\section{DISCUSSION}

The reason why certain rumen ciliates such as Ophryoscolex and Epidinium are less readily established in young lambs and kids than other ciliate species is not known, nor is it clear why Ophryoscolex disappears from populations in older animals. This latter change does not appear to be caused by the direct action of Polyplastron since Ophryoscolex is regularly a component of type A populations and this is confirmed by the observation that Ophryoscolex will also disappear from a population with the type B organism Eudiplodinium as the only other large Ophryoscolecid. It seems that this variation in the stability and ease of establishment of Ophryoscolex in older animals (see p. I82) may be a demonstration of differences between individual hosts (cf. Eadie, $1962 a$ ). It is possible that the failure of Polyplastron to become dominant just before the death of sheep 209 may be a further example of features of the host or its bacterial flora affecting the ciliate population rather than unusual features of the ciliate population per se being the cause of an apparent anomaly.

The disappearance of Ophryoscolex from a population which includes Epidinium differs from the above noted instability of Ophryoscolex in that it is directly related to the presence of Epidinium. However, failure to reproduce the antagonism in a vivar indicates that the speed of the effect is not as great as when Polyplastron becomes dominant by predation. The cause of the antagonism is not yet clear but it seems that other factors such as food competition, rather than predation, may be involved.

The observed reduction in size of caudal processes in a developing Ophryoscolex population shows that these organelles are not a reliable taxonomic feature. A reduction in the caudal processes of Entodinium caudatum has been noted by Coleman (I963) in cultures of that species and a similar, apparently permanent, reduction has been seen in E. caudatum maintained as the major ciliate species in an isolated sheep. Several years after the species had been established caudal processes were very rarely seen and did not redevelop when the population was transferred by inoculation to a young ciliate-free lamb.

Predation by Polyplastron has been shown to differ from the 'accidental' predation previously noted by Lubinsky (I957) in that it leads to the complete removal from the population of those species which form the prey. The reasons why some species should be eliminated while others remain, although occasionally ingested, are not known. The regular appearance of predatory or cannibalistic Polyplastron in sheep ciliate populations suggests that these features are common to all Polyplastron and are not a limited racial characteristic. This is also indicated by the apparent return of large organisms to a smaller size. The stimulus to predation in Polyplastron is still not clear, but as in the case of cannibalism in Stentor (Tartar, 196I) it is due neither to over-feeding nor starvation, nor is it merely caused by a lack of protein. Predation is more effective than cannibalism in keeping the protozoa alive. The ingestion of ground-nut meal and barley leaf protein, though not preventing predation within a vivar, leads to an in- 
creased rate of utilization of carbohydrate. On the other hand predation does not markedly increase the carbohydrate utilization, which suggests that the ingested organisms are a less satisfactory protein source than the other proteins tested. Predatory 'activity' appears to be increased by previous predation. The significance to the organisms of predation is difficult to assess. As noted previously (Eadie, 1962 b) the numbers of organisms tend to drop after a 'take-over' in sheep and this reduction in numbers could be due to a slower division rate or to cannibalism. There is no reason to believe that cannibalism is widespread amongst Polyplastron in vivo but it does occur at the onset of food shortage and under those conditions would have the effect of reducing the food requirements of the population, and perhaps ensuring survival of the species. A similar explanation does not, however, explain predation by Polyplastron. While predation of an original type B population leaves Polyplastron with more food and space per organism, there is no reason to believe that food supply was in fact limiting before predation began. Under the same conditions other type B organisms would have been successfully established in the population.

The complete and rapid elimination of a species from a particular habitat because of direct interaction with another species does not appear to be very common in nature (Elton, 1958; Andrewartha, 1961). The present observations are probably most nearly comparable with the observed relationship between the larvae of two species of blowflies, Lucilia sericata and Chrysomyia albiceps, cultured on meat (Ullyett, 1950). Though both species were living on the same food, of which there was an ample supply, C. albiceps first killed the larvae of Lucilia in preference to ingesting the food and thus Lucilia was eliminated.

Arising from the present work the most obvious question still to be answered is what in the course of evolution has saved type B organisms from extinction. There must be numerous transfers of ciliates by mouth to mouth contact between host animals yet type B and type A populations are both common. Clearly conditions under which Polyplastron fail to become dominant must occur quite frequently but the nature of these conditions has not yet been determined. In cattle and in goats largesized Eudiplodinium have been observed and these appear to be associated with a tendency for a change from type A to type B in cattle (see p. I83). Large-sized Eudiplodinia have not been produced experimentally in a vivar with sheep as 'host' but work with cattle as 'host' is planned. The question of large size and its relationship to food and predation is being further investigated.

I am indebted to Dr T. Woodham for the samples of barley-leaf protein and groundnut meal. My thanks are due to Dr P. N. Hobson for much helpful discussion and for his assistance, together with that of Dr L. Fina, in the design of the vivar apparatus, and to $\mathrm{Mr} \mathrm{W}$. Shand for his continued and invaluable assistance throughout this work.

\section{RIFHRECES}

Abou Akkada, A. R. \& Howard, B. H. (1960). The biochemistry of rumen protozoa. 3. The carbohydrate metabolism of Entodinium. Biochem. $J .76,445$.

Andrewartha, H. G. (1961). In Introduction to the Study of Animal Populations. Chicago and London: University of Chicago Press.

Brown, T. J. (1964). The inter-relationship of two dominant ciliate species in an activated sludge plant. J. Protozool. I r, suppl. p. 38.

Coleman, G.S. (1963). The growth and metabolism of rumen ciliate protozoa. Symp. Soc. gen. Microbiol. I3, 298. 
Dawson, J. A. (19I9). An experimental study of an amicronucleate Oxytricha. I. Study of the normal animal, with an account of cannibalism. J. exp. Zool. 29, 473.

DogIEL, V. A. (1947). The phylogeny of the stomach-infusorians of ruminants in the light of palaeontological and parasitological data. Quart. J. micr. Sci. 88, 97.

Duckworth, J. \& Woodham, A. A. (I96I). Leaf protein concentrates. I. Effect of source of raw material and method of drying on protein value for chicks and rats. J. Sci. Fd Agric. 12, 7.

EAdIE, J. M. (I962a). The development of rumen microbial populations in lambs and calves under various conditions of management. J. gen. Microbiol. 29, 563.

EADIE, J. M. (I962b). Inter-relationships between certain rumen ciliate protozoa. J. gen. Microbiol. 29, 579.

EAdIE, J. M. \& OXFORD, A. E. (I955). Factors involved in the production of a novel kind of derangement of storage mechanism in living holotrich ciliate protozoa from sheep rumen. $J$. gen. Microbiol. 12, 298.

Elton, C. (1958). In The Ecology of Invasions. London: Methuen and Co. Ltd.

Fina, L. R., Keith, C. L., Bartley, E. E., Hartman, P. A. \& Jacobson, N. L. (1962). Modified in vivo artificial rumen (vivar) techniques. J. Anim. Sci. 2I, 930.

Geler, J. von (I925). Über den Kannibalismus bei Stentoren. Arch. Protistenk. 52, 404.

Giese, A. C. (1938). Cannibalism and gigantism in Blepharisma. Trans. Am. microsc. Soc. 57, 245.

Giese, A. C. \& Alden, R. H. (I938). Cannibalism and giant formation in Stylonychia. J. exp. Zool. $78,117$.

LUBINSKY, G. (I957). Note on the phylogenetic significance of predatory habits in the Ophryoscolecidae (Ciliata: Oligotricha). Can. J. Zool. 35, 579.

Padmavathi, P. B. (196I). Giant cannibals in Blepharisma undulans (Protozoa: Ciliata). Arch. Protistenk. I05, 34I.

Proctor, V. W. (I 957). Studies of algal antibiosis using Hematococcus and Chlamydomonas. Limnol. Oceanogr. 2, 125.

RICE, T. R. (I954). Biotic influences affecting population growth of planktonic algae. Fishery Bull. Fish Wildl. Serv. U.S. 54, 227.

TARTAR, V. (I96r). In The Biology of Stentor. International Series of Monographs on Pure and Applied Biology, Division Zoology, London: Pergamon Press.

Ullyetr, G. C. (1950). Competition for food and allied phenomena in sheep blowfly populations. Phil. Trans. R. Soc. $\mathbf{B}$ 234, 77.

\section{EXPLANATION OF PLATES}

\section{Plate I}

Fig. I. The components of a vivar chamber-for explanation see text.

Fig. 2. The method by which the vivars are suspended in the rumen-for explanation see text.

Plate 2

All the organisms were fixed with formalin and were unstained.

Fig. I-fig. 7 magnified $\times 105$

Fig. I. Eudiplodinium maggii from goat G 108 prior to the addition of Polyplastron multivesiculatum to the population.

Fig. 2. Unusually large Eudiplodinium from goat G 108 after the addition of Polyplastron and 5 days before the change-over of population. Ophryoscolex is also seen.

Fig. 3. Large, densely filled dividing, Polyplastron from the same sample as Fig. 2. Ophryoscolex and a 'normal'-sized Eudiplodinium are also seen.

Fig. 4. Densely filled Polyplastron from lamb 222 at the time of a change-over of population. The type $\mathbf{B}$ organism Epidinium is also seen and a number of entodinia.

Fig. 5. Sample from a vivar at the time of a change-over of population. Densely filled Polyplastron from 6108 and a 'normal'-sized Eudiplodinium from sheep 95 are seen.

Fig. 6. Polyplastron from 683 before a.m. feed. Cannibalism is seen in organism $a$. Internal structure, e.g. the two large skeletal plates, can be seen in $b$ and $c$.

Fig. 7. Sample from a vivar taken just after a change-over of population. Polyplastron which have become misshapen through 'chewing' by other Polyplastron are seen. 
Plate I
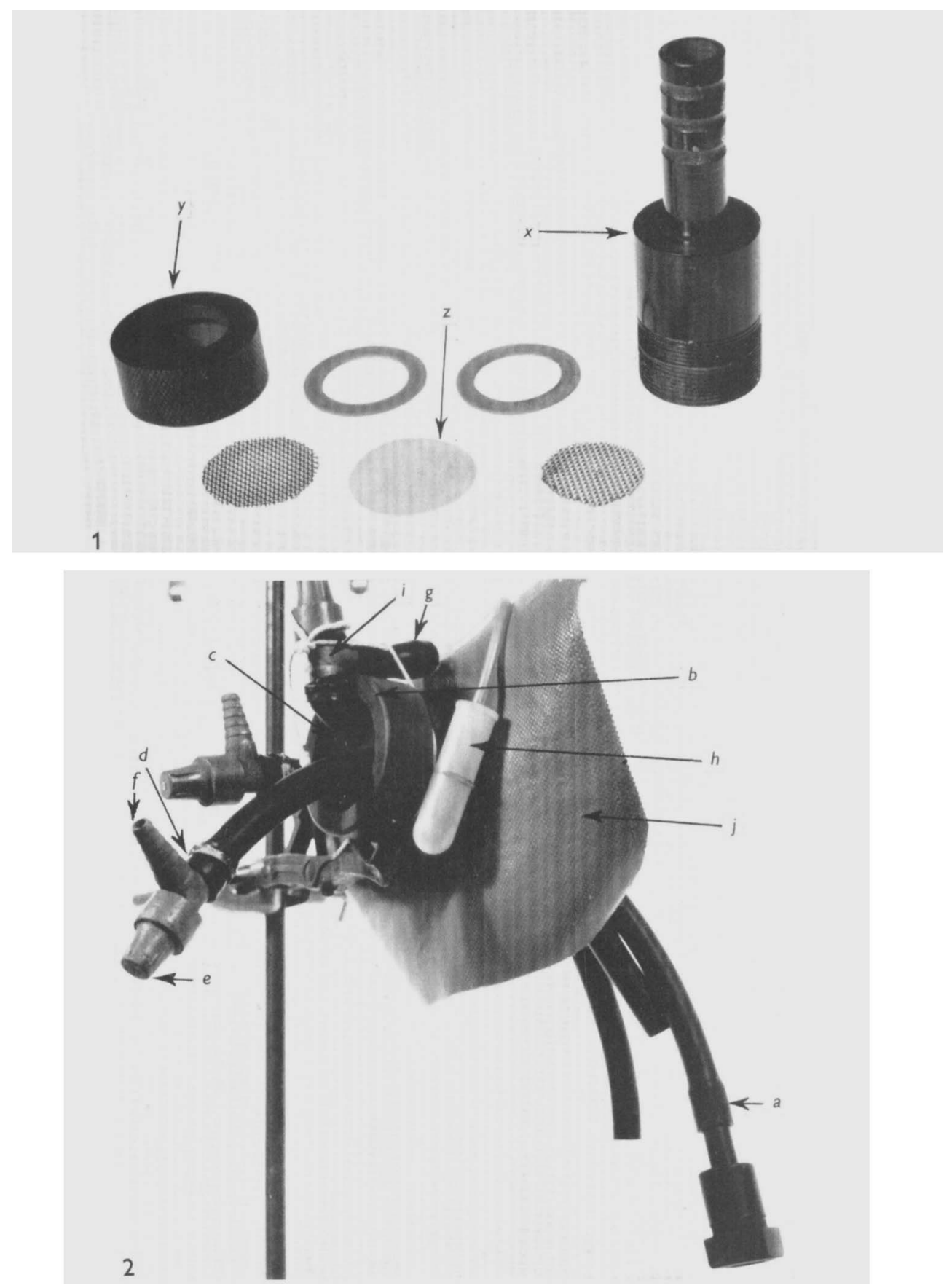

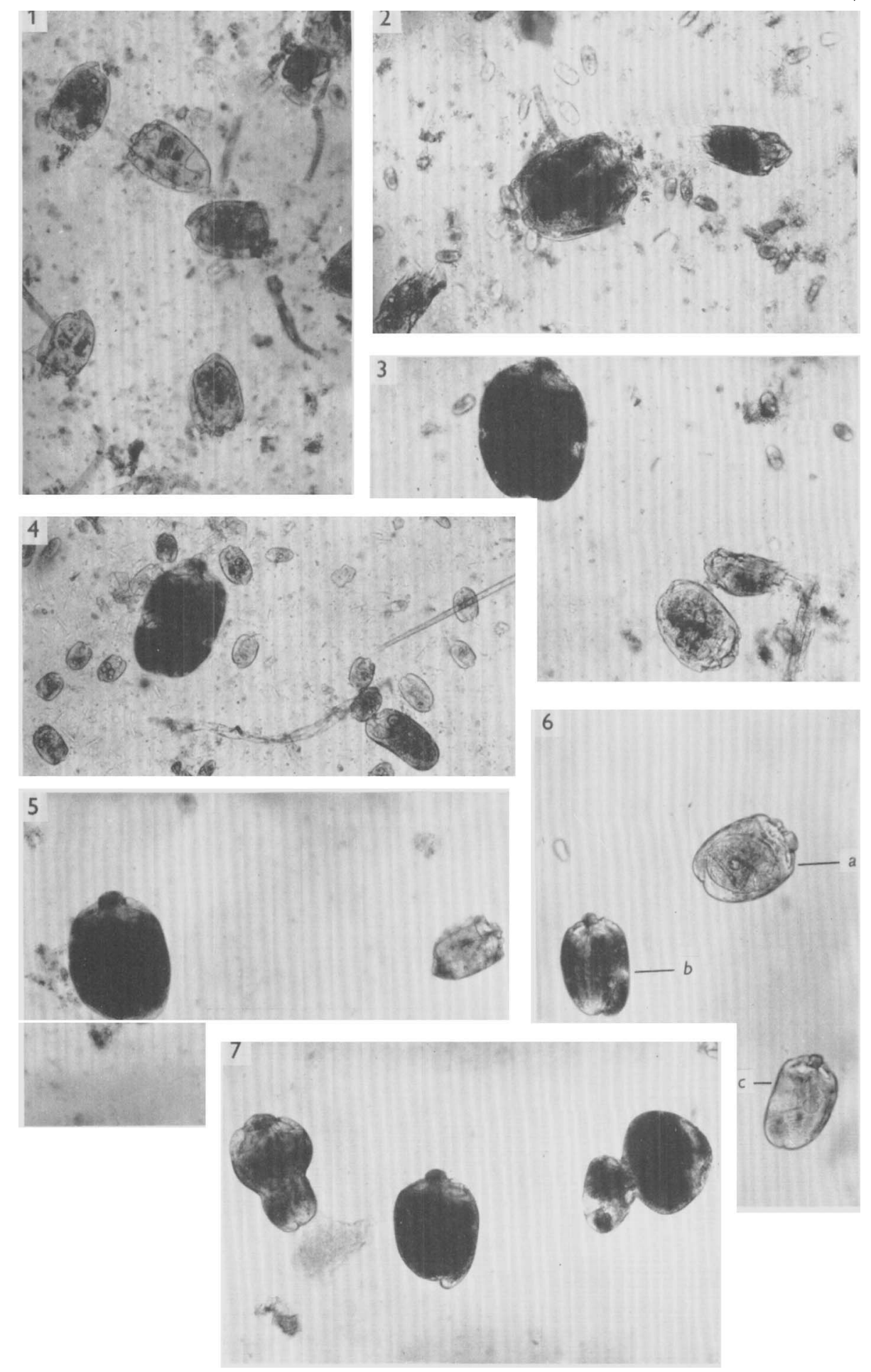